\title{
Source Identification and Noise Reduction of a Reciprocating Compressor; a Case History
}

\author{
N. Bert Roozen \\ Philips Applied Technologies, High Tech Campus 7, 5656AE Eindhoven, The Netherlands \\ Eindhoven University of Technology, P.O. Box 513, 5600MD Eindhoven, The Netherlands \\ Jozèf van den Oetelaar \\ GEA Grasso, Parallelweg 27, 5223AL 's Hertogenbosch, The Netherlands
}

\author{
Alex Geerlings, Theon Vliegenthart \\ Philips Applied Technologies, High Tech Campus 7, 5656AE Eindhoven, The Netherlands
}

(Received 1 April 2009; accepted 8 May 2009)

\begin{abstract}
A noise source can be very complex in nature. In noise control engineering an essential first step is to identify the strongest contributing noise sources. This paper discusses a practical case history, in which a series of measurement techniques was applied to a reciprocating compressor to identify the strongest sound source. In addition numerical prediction methods were used to give direction towards a lower noise design of the compressor. Structural modifications to the compressor are discussed, leading to a significant reduction of the noise levels. The sound power level of the reciprocating compressor was reduced by $5 \mathrm{~dB}$. Moreover, from a perceptual point of view, customers experience the sound as more robust and more pleasant as well, probably because of shifting frequencies.
\end{abstract}

\section{INTRODUCTION}

During the development of the Grasso V700 compressor a 4 cylinder prototype was created consisting of bended steel plates welded together, as shown in Fig. 1. The plating for the crankcase had a thickness of $15 \mathrm{~mm}$, whilst the cylinder heads were constructed from $8 \mathrm{~mm}$ thick steel plates for the bended parts and $15 \mathrm{~mm}$ plating for the end grains. The material used was steel S355J2+N. ${ }^{1}$ The new assembly is straightforward, allowing a more efficient production as compared to other compressor concepts. Besides, separated cylinder heads have major advantages in terms of thermodynamic behavior. However, it turned out that the steel plated compressor produced an unwanted increase in noise of approximately $6 \mathrm{~dB}$ compared to its predecessor, the Grasso 412 compressor, which used a steel pipe welded cylinder head.

This paper describes a practical case history of the acoustic analysis and redesign of the compressor to restore former noise levels. Design directions were based on in-situ measurements and numerical simulations.

The paper is organized as follows. It starts in Section 2 with an experimental noise analysis, including sound pressure level measurements and sound intensity measurements of the complete compressor to determine the most dominant frequency bands. This is followed by a partial noise source analysis by means of a surface intensity measurement technique to identify the most dominant part of the machine contributing to the dominant frequency band. After that a modal analysis measurement and a modal analysis simulation are presented of the dominant part of the compressor. Finally, in Section 3 a structural modification of the compressor is discussed. Its implementation was acoustically validated by means of a sound intensity measurement of the complete compressor, confirming a significant noise reduction. In Section 4 the conclusions are given.

\section{NOISE ANALYSIS}

\subsection{Compressor harmonics}

For a compressor operating at $1200 \mathrm{rpm}$, the fundamental frequency of the noise spectrum corresponds to $1200 / 60=20$ Hz. A sound pressure level measurement was performed. Figure 2 shows that the compressor noise was dominated by the fundamental rotational frequency and its harmonics. Only the fundamental and (many) harmonic frequencies of the rotation speed contribute to the spectrum, covering the whole audible frequency range. Therefore, shifting operational speed in order to avoid structural resonances is obviously not an option. Generally speaking, excitation forces are rarely purely sinusoidal in form, so that harmonics appear naturally as can be explained by Fourier series expansion. In this specific case of a compressor it is caused by the fact that the compression in the cylinder is far from smooth, causing an abrupt increase of the pressure inside the cylinder. Moreover, valve noise also attributes to the noise spectrum, which contains many harmonics because of its impulse repetitive character. Excitation due to discrete harmonics is typical in many structural acoustic cases.

Though the acoustic noise consists only of discrete frequencies, the dynamics of the structural components of the compressor play an important role. Resonant behavior of structural components of the compressor may increase the structural response. However, the presence of structural resonances is now more obscured compared to cases in which a mechanical system is excited by a more broadband spectrum. 